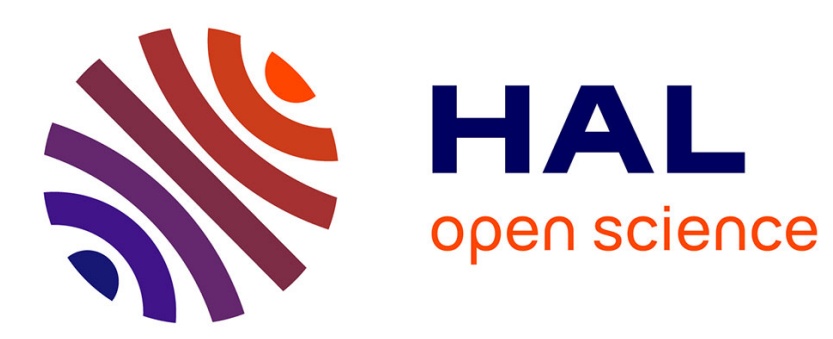

\title{
Première observation de l'anthracnose de la luzerne dans le Maroc oriental
}

\author{
Bun Meng Troeung, Huguette Gosset
}

\section{To cite this version:}

Bun Meng Troeung, Huguette Gosset. Première observation de l'anthracnose de la luzerne dans le Maroc oriental. Agronomie, 1987, 7 (5), pp.361-363. hal-00885002

\section{HAL Id: hal-00885002 https://hal.science/hal-00885002}

Submitted on 1 Jan 1987

HAL is a multi-disciplinary open access archive for the deposit and dissemination of scientific research documents, whether they are published or not. The documents may come from teaching and research institutions in France or abroad, or from public or private research centers.
L'archive ouverte pluridisciplinaire HAL, est destinée au dépôt et à la diffusion de documents scientifiques de niveau recherche, publiés ou non, émanant des établissements d'enseignement et de recherche français ou étrangers, des laboratoires publics ou privés. 


\section{Première observation de l'anthracnose de la luzerne dans le Maroc oriental}

Bun Meng TROEUNG \& Huguette GOSSET

Département de Biologie, Faculté des Sciences, Université Mohammed-I ${ }^{e r}$, Oujda, Maroc

Un inventaire des principales maladies de la Luzerne en culture irriguée met en évidence l'importance de l'anthracnose dans le Maroc oriental. Les agents responsables ont été isolés et inoculés sur luzerne en chambre de culture; les symptômes typiques ont été reproduits. Il s'agit de Colletotrichum trifolii Bain et Essary auquel la variété « Moapa », largement encouragée dans la région, s'avère sensible, et de $C$. destructivum O'Gara, faiblement pathogène. Des tests de résistance variétale de différents Medicago sont en cours.

Mots clés additionnels : Colletotrichum trifolii, Colletotrichum destructivum, Medicago.

A survey of the main diseases of lucerne in irrigated fields in Eastern Morocco showed the importance of anthracnose. Two pathogens were isolated and reinoculated into lucerne in a growth chamber ; typical symptoms were reproduced. The disease was due to Colletotrichum trifolii, to which cv. Moapa much recommended in the region is very susceptible, and to $C$. destructivum, which is weakly pathogenic. Tests of resistant cultivars are in progress.

Additional key words : Colletotrichum trifolii, Colletotrichum destructivum, Medicago.

\section{INTRODUCTION}

L'anthracnose de la luzerne (Medicago sativa L.), due à Colletotrichum spp. n'est pas signalée dans les inventaires des organismes pathogènes et saprophytiques des plantes au Maroc (RIEUF, 1971 ; anonyme, 1965 ; RAYNAL, 1977a). En revanche, cette maladie est notée en différents continents : Amérique du Nord et du Sud, Afrique du Sud, Océanie et Europe, en particulier en Europe méditerranéenne (RAYNAL, 1977a ; RAYNAL, 1986).

Nous avons observé la maladie dans les luzernières irriguées de plusieurs exploitations du Maroc oriental (régions d'Oujda et de Berkane), de décembre 1984 à décembre 1986. La variété utilisée couramment dans cette région est la variété synthétique "Moapa» (BOLTON, 1962). Le rythme de coupe, de trois semaines en été, de un mois à un mois et demi en hiver, permet d'obtenir des rendements élevés.

Les plantes atteintes d'anthracnose se signalent par le dessèchement des tiges dont l'extrémité se recourbe en crosse. Une observation plus attentive montre de minuscules points et des taches nécrotiques allongées, de couleur brun foncé, disposés à la base de la tige. Les nécroses, en s'étendant, deviennent coalescentes et aboutissent aux lésions chancreuses qui, finalement, ceinturent la tige, entraînant le dessèchement complet des repousses. L'anthracnose semble représenter les attaques nécrotiques les plus fréquentes des tiges. Signalons en outre que les pourritures de racines, dues à Fusarium oxysporum Schlecht., entraînent un jaunissement généralisé des parties aériennes observable principalement en hiver, et que les feuilles sont communément attaquées par Peronospora aestivalis. Syd. Apud. Gaüm., Uromyces striatus Schroet. var. medicaginis (Pass.) Arth. et Pseudopeziza medicaginis (Lib.) Sacc.

L'anthracnose peut être sévère dès la deuxième année. Alors que la pérennité des luzernes saines irriguées est de 5 ans dans la région d'Oujda, les agriculteurs sont amenés à retourner leurs champs malades dès la troisième année car, même si les plants attaqués 
redémarrent grâce à de nouvelles repousses, les rendements sont affectés et les nécroses constituent autant de portes d'entrée pour les parasites opportunistes.

On remarque que l'ampleur de la maladie est particulièrement grande en novembre et décembre, à une époque où la moyenne des températures maximales dans la région prospectée avoisine $25^{\circ} \mathrm{C}$. En été, malgré l'irrigation, les températures semblent trop fortes pour que la maladie se développe.

\section{MATÉRIEL ET MÉTHODES}

\section{A. Isolement et détermination de l'agent pathogène}

Sur certaines lésions, des acervules sont visibles à la loupe, sous forme de coussinets hérissés de soies brunes. Ce caractère permet d'identifier le genre Colletotrichum dont plusieurs espèces s'observent dans les cas d'anthracnose (RAYNAL, 1977b).

En vue d'une détermination plus précise, des prélèvements sont pratiqués dans 3 exploitations de la banlieue Est d'Oujda, distantes de 1 à $3 \mathrm{~km}$ les unes des autres. Des fragments de tiges avec lésions chancreuses sont mis à incuber en chambre humide. Après 3 ou 4 jours, les acervules sporulent. Les gelées sporifères, d'un rose passé, sont prélevées et déposées en boîtes de Petri, par étalement, à la surface d'un milieu nutritif (potato dextrose agar) préparé selon la technique de PAULy (1973). A $25^{\circ} \mathrm{C}$, les spores germent en donnant un mycélium blanc, légèrement rosé, qui devient vert olive à partir du $3^{\mathrm{e}}$ jour.

Le thalle obtenu après repiquage présente des contours réguliers. D'abord ras, il se couvre ensuite d'un mycélium aérien blanc, discrètement floconneux ; certains thalles développent des secteurs où le mycélium aérien est plus développé. Quelques acervules sont déjà observables au $7^{\mathrm{e}}$ jour; elles deviennent ensuite très abondantes dans les parties âgées du thalle. La croissance diamétrale journalière des 3 isolats, de $3,5 \mathrm{~mm}$ (moyenne de 60 mesures) au $7 \mathrm{e}$ jour, est relativement lente. Les spores, hyalines, droites, présentent des extrémités arrondies et une taille variable pour un même isolat: $10-16 \mu \mathrm{m} \times 3-5 \mu \mathrm{m}$. L'ensemble de ces caractères conduit à l'identification, dans les 3 cas, de Colletotrichum trifolii Bain \& Essary (RAYNAL, 1977b).

Fréquemment, une autre espèce de Colletotrichum est isolée. Sa morphologie présente quelques différences, comparée à celle de l'espèce précédente. Le mycélium jeune est blanc grisâtre ; même âgé, il demeure ras. 11 porte dès le $3^{\text {e }}$ jour des acervules de petite taille, dispersées très régulièrement sur l'ensemble du thalle; les gelées sporifères sont crème. La croissance diamétrale journalière est de $4,3 \mathrm{~mm}$ (moyenne de 20 mesures) au $7 \mathrm{e}$ jour. Les spores, hyalines, droites ou légèrement arquées, ont des extrémités arrondies, mesurent $15-19 \mu \mathrm{m} \times 3-3,5 \mu \mathrm{m}$. Ces caractères sont ceux de Colletotrichum destructivum O'Gara.

\section{B. Inoculations artificielles}

Le test du pouvoir pathogène des isolats de $C$. trifolii est effectué sur de jeunes luzernes de la variété «Moapa ». Pour chaque isolat, 200 plantules, obte- nues à partir de graines désinfectées à l'eau de javel et prégermées, sont repiquées dans 4 bacs remplis de sable stérilisé à la chaleur sèche $\left(180^{\circ} \mathrm{C}\right)$, et arrosées avec une solution nutritive.

Les plantes âgées de 21 jours sont inoculées artificiellement selon la technique décrite par RAYNAL (1977b), par pulvérisation d'une suspension de $3.10^{5}$ spores $/ \mathrm{ml}$. Les plantes sont ensuite maintenues à 100 p. 100 d'humidité relative sous une housse en matière plastique transparente, éclairée $18 \mathrm{~h} / \mathrm{j}$ à l'aide de tubes fluorescents (14 tubes pour $1,2 \mathrm{~m}^{2}$ ). Les températures sont de $31{ }^{\circ} \mathrm{C}$ le jour et de $21{ }^{\circ} \mathrm{C}$ la nuit.

Dans les jours qui suivent l'inoculation, les taches nécrotiques apparaissent sur les pétioles et les tiges. D'abord ponctuelles, elles évoluent en taches allongées, lesquelles entourent progressivement la tige, comme cela se produit au champ. Elles entraînent le jaunissement, puis le dessèchement des organes aériens au-dessus des zones d'attaque.

Les symptômes observés le $11^{\mathrm{e}}$ jour sont notés selon l'échelle citée par RAYNAL $(1977 b)$. Les isolats $C_{09}$, $\mathrm{C}_{11}$ et $\mathrm{C}_{12}$ de $C$. trifolii, obtenus à partir des échantillons récoltés dans les trois exploitations sus-citées, ont manifesté une forte agressivité. Les moyennes des notes attribuées sont respectivement 4,$3 ; 3,9 ; 4,3$. Les pourcentages de plantes résistantes (notées 1 et 2 ) sont respectivement de 12,21 et 12 p. 100 tandis que les plantes mortes (notées 5) représentent 71,62 et 73 p. 100 des plantes inoculées.

Le pouvoir pathogène de $C$. destructivum est testé dans des conditions identiques à celles décrites pour C. trifolii. Les nécroses ne représentent pas la même gravité : ce sont essentiellement les pétioles qui sont atteints, éventuellement les feuilles. Au moment de la notation, 2 p. 100 seulement des plantes sont mortes et les acervules ne sont pas encore observables.

\section{DISCUSSION-CONCLUSION}

L'une et l'autre espèce sont réisolables à partir des plantes inoculées. Toutefois, les tests cités plus haut prouvent que $C$. trifolii manifeste une forte agressivité, alors que $C$. destructivum ne présente que peu d'agressivité. Le faible pouvoir pathogène de cette dernière espèce ne peut expliquer l'ampleur des dégâts observés au champ. Il est donc permis d'affirmer que C. trifolii est le principal responsable de l'anthracnose de la luzerne qui sévit dans le Maroc Oriental.

Une seconde remarque s'impose. La variété "Moapa ", largement implantée dans la région, se montre particulièrement sensible à l'anthracnose, au champ et en chambre de culture. Nous nous proposons de publier prochainement les résultats d'expérimentations en cours concernant la résistance variétale à l'anthracnose de différents cultivars de Medicago sativa et de Medicago sauvages.

Reçu le 20 décembre 1986. Accepté le 15 janvier 1987.

\section{REMERCIEMENTS}

I.es auteurs remercient Monsieur G. RAYNAL pour ses conseils et ses critiques lors du deroulement de ce travail. 


\section{RÉFÉRENCES BIBLIOGRAPHIQUES}

Anonyme, 1965. Les Cultures Fourragères Irriguées au Maroc. Coll. Technique et Productions agricoles, I.N.R.A., Rabat.

Bolton J. L., 1962. Alfalfa: Botany, Cultivation and Utilization. Interscience Publishers Inc., N.Y., p. 140.

Pauly G., 1973. Les Colletotrichum de la luzerne en France : Systématique, biologie, pouvoir pathogène de quelques souches. D.E.A. Fac. Sci. Orsay, 58 p.

Raynal G., 1977a. Maladies cryptogamiques des légumineuses alimentaires, fourragères et industrielles. $5^{\mathrm{c}}$ j. Phytiât. Phytoph. Circum-méditerr., Rabat, p. 251-268.
Raynal G., 1977b. Comparaison, en contaminations artificielles, des pouvoirs pathogènes des Colletotrichum isolés en France sur la luzerne. Ann. Phytopathol., 9 (2), 193-203.

Raynal G., 1986. Les maladies de la luzerne en Europe, p. 7-14. In : La production de fourrage et de semences de luzerne en Europe. Maladies, ravageurs et variétés. EUCARPIA «Medicago sativa". I.N.R.A. Ed.

Rieuf P., 1971. Parasites et saprophytes des plantes au Maroc. Cahiers de la recherche agronomique. Rabat. 29, p. 358-463. 